\title{
The Current State of Pediatric Hospital Medicine Fellowships: A Survey of Program Directors
}

\author{
Neha H. Shah, MD, MPH ${ }^{1 \star}$, Hai Jung H. Rhim, MD, MPH², Jennifer Maniscalco, MD, MPH${ }^{3}$, \\ Karen Wilson, MD, $\mathrm{MPH}^{4}$, Caroline Rassbach, $\mathrm{MD}^{5}$
}

\begin{abstract}
${ }^{1}$ Division of Hospitalist Medicine, Children's National Health System, Washington, DC; 'Division of Hospital Medicine, Children's Hospital at Montefiore, Bronx, New York; 'Division of Hospital Medicine, Children's Hospital Los Angeles, Los Angeles, California; ' $D i v i s i o n$ of Hospital Medicine, Children's Hospital Colorado, Aurora, Colorado; ${ }^{5}$ Division of Pediatric Hospital Medicine, Stanford University, Palo Alto, California.
\end{abstract}

BACKGROUND: Pediatric hospital medicine (PHM) fellowship programs have grown rapidly over the last 20 years and have varied in duration and content. In an effort to standardize training in the absence of a single accrediting body, PHM fellowship directors now meet annually to discuss strategies for standardizing and enhancing training.

OBJECTIVES: To explore similarities and differences in curricular structure among PHM fellowship programs in an effort to inform future curriculum standardization efforts.

METHODS: An electronic survey was distributed by e-mail to all PHM fellowship directors in April 2014. The survey consisted of 30 multiple-choice and short-answer questions focused on various curricular aspects of training developed by the authors.

RESULTS: Twenty-seven of 31 fellowship programs (87\%) responded to the survey. Duration of most programs was 2 years $(63 \%)$, with 6, 1-year programs (22\%) and $4(15 \%)$ 3 -year programs making up the remainder. The average amount of clinical time among programs was 50\% (range approximately $20 \%-65 \%$ ). In addition to general inpatient pediatric service time, most programs require other clinical rotations. The majority of programs allow fellows to bill independently for their services. Most programs offer certificate courses, courses for credit or noncredit courses, with 11 programs offering masters' degrees. Twenty-one (81\%) programs provide a scholarship oversight committee for their fellows. Current fellows' primary areas of research are varied.

CONCLUSION: Though variability exists regarding program length, clinical composition, and nonclinical offerings, several common themes emerged that may help inform the development of a standard curriculum for use across all programs. This information provides a useful starting point if pediatric hospital medicine obtains formal subspecialty status. Journal of Hospital Medicine 2016;11:324-328. (c) 2016 Society of Hospital Medicine
Pediatric hospital medicine (PHM) fellowship programs came into existence approximately 20 years ago in Canada, ${ }^{1}$ and since that time the number of programs in North America has grown dramatically. The first 3 PHM fellowship programs in the United States were initiated in 2003, and by 2008 there were 7 active programs. Just 5 years later in 2013, there were 20 fellowship programs in existence. Now, in 2015, there are over 30 programs, with several more in development. The goal of postresidency training in PHM is to improve the care of hospitalized children by training future hospitalists to provide high-quality, evidence-based clinical care and to generate new knowledge and scholarship in areas such as clinical research, patient safety and quality improvement, medical education, practice management, and patient

*Address for correspondence and reprint requests: Neha $\mathrm{H}$. Shah, MD, Division of Hospitalist Medicine, Children's National Health System, 111 Michigan Avenue NW, Suite M-4800, Washington DC, 20010; Telephone: 202-476-4835; Fax: 202-476-3732;

E-mail: nshah@childrensnational.org

Additional Supporting Information may be found in the online version of this article.

Received: July 20, 2015; Revised: October 23, 2015; Accepted: December 4, 2015

2016 Society of Hospital Medicine DOI 10.1002/jhm.2571

Published online in Wiley Online Library (Wileyonlinelibrary.com). outcomes. $^{2}$ Many pediatric hospitalists want to be able to perform research or quality improvement, but feel that they lack the time, skills, resources, and mentorship to do so. ${ }^{3}$ To date, fellowship-trained hospitalists have a demonstrated track record of contributing to the body of literature that is shaping the care of hospitalized children. ${ }^{4,5}$

At present, PHM is not a recognized subspecialty of the American Board of Pediatrics (ABP) and therefore does not fall under the purview of the Accreditation Council of Graduate Medical Education (ACGME), leading to concern from some about the variability in depth and breadth of training across programs. ${ }^{1}$ The development and publication of the PHM Core Competencies in 2010 helped define the scope of practice of pediatric hospitalists and provide guidelines for training programs, specifically with respect to clinical and nonclinical areas for assessment of competency. ${ }^{6}$ Furthermore, studies of early career hospitalists have identified areas for future fellowship curriculum development, such as core procedural skills, quality improvement, and practice management. ${ }^{7}$

In an effort to address training variability across programs, PHM fellowship directors (FDs) have come together as an organized group, first meeting in 2008, with the primary goal of defining training standards 


\section{Variability in Weeks on Inpatient General Pediatrics Service}

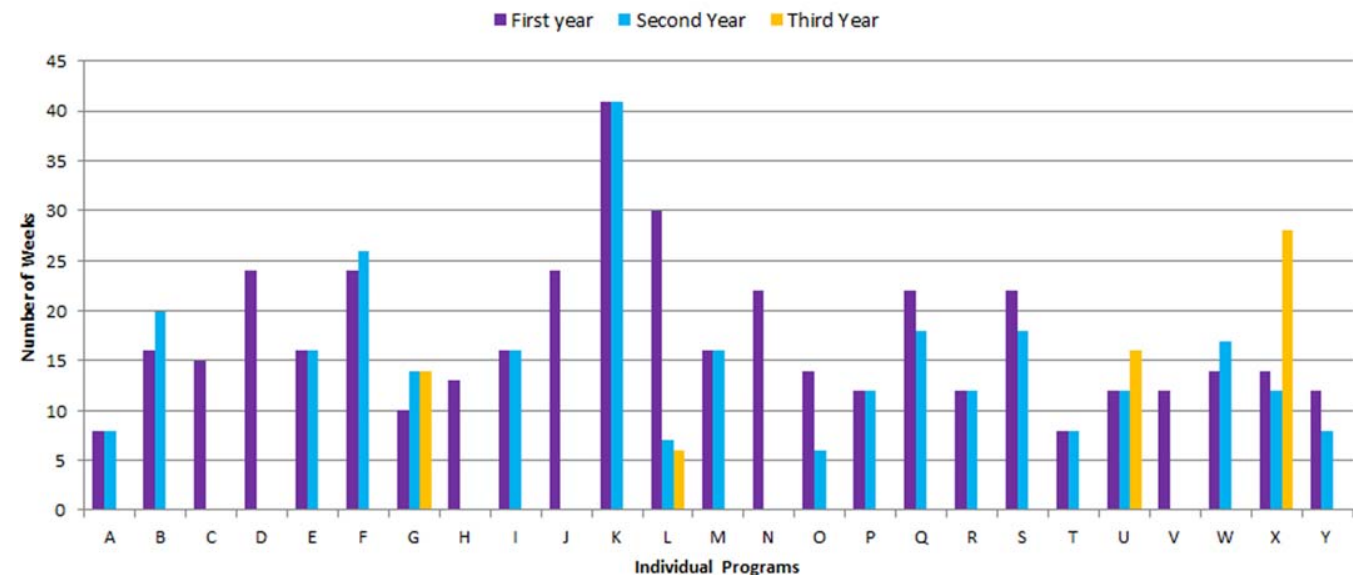

FIG. 1. Variability in weeks of inpatient general pediatrics service.

and sharing curricular resources. Annual meetings of the FDs, sponsored by the American Academy of Pediatrics Section on Hospital Medicine (AAP-SOHM), began in 2012. A key objective of this annual meeting has been to develop a standardized fellowship curriculum for use across programs as well as to determine gaps in training that need to be addressed. During this process, we have received input from key stakeholders including community hospitalists, internal medicine-pediatrics hospitalists, and the PHM Certification Steering Committee, which organized the application for subspecialty certification to the ABP. To inform this process of curriculum standardization, we fielded a survey of PHM fellowship directors. The purpose of this article is to summarize the current curricula, operations, and logistics of PHM fellowship programs.

\section{METHODS}

This was a cross-sectional study of 31 PHM fellowship programs across the United States and Canada in April 2014. Inclusion criteria included all pediatric fellowships that were self-identified to the AAP-SOHM as providing a hospital medicine fellowship option. This included both PHM fellowships as well as academic general pediatric fellowships with a hospitalist track. A web-based survey (SurveyMonkey, Inc.) was distributed by e-mail to the FDs at the 31 training programs (see Supporting Information in the online version of this article). To enhance content validity of survey responses, survey questions were designed using an iterative consensus process among the authors, who included junior and senior FDs and represented the 2014 annual FD meeting planning committee. Items were created to gather feedback on the following key areas of PHM fellowships: program demographics, types of required and elective clinical rotations, graduate coursework offerings, amount of time spent in clinical activities, fellow billing practices, and description of fellows' research activities. The survey consisted of 30 multiple-choice and short-answer ques- tions. Follow-up e-mail reminders were sent to all FDs 2 weeks and 4 weeks after the initial request was sent. Survey completion was voluntary, and no incentives were offered. The study was determined to be exempt by the Stanford University Institutional Review Board. Data were summarized using frequency distributions. No subgroup comparisons were made.

\section{RESULTS}

Program directors from 27/31 (87\%) PHM fellowship programs responded to the survey; 25 were active programs, and 2 were under development. Responding programs represented all 4 major regions of the country and Canada, with varying program initiation dates, ranging from 1997 to 2013.

\section{Program Demographics}

The duration of most programs (17/27) was 2 years $(63 \%)$, with $6(22 \%)$ 1-year programs and $4(15 \%) 3$ year programs making up the remainder. Four programs described variable lengths, which could be tailored based on the fellow's individual interest. Two of the programs are 2 years in length, but offer a 1-year option for fellows who wish to focus on enhancing clinical skills without an academic focus. The other 2 programs are 2 years in length, but will offer an extension to a third year for those pursuing a graduate degree.

\section{Fellow Clinical Activities}

The average amount of total clinical time (weeks on service) across responding programs was 50\% (range, $20 \%-65 \%$ ). When looking specifically at time on the inpatient general pediatric service, number of weeks varied by year of training and by institution, with 12 to 41 weeks in the first year of fellowship, 6 to 41 weeks in the second year of fellowship, and 6 to 28 weeks in the third year of fellowship (Figure 1). Though the range is large, on average, fellows spend 17 weeks on inpatient general pediatrics service during each year of training. Of note, the median number 


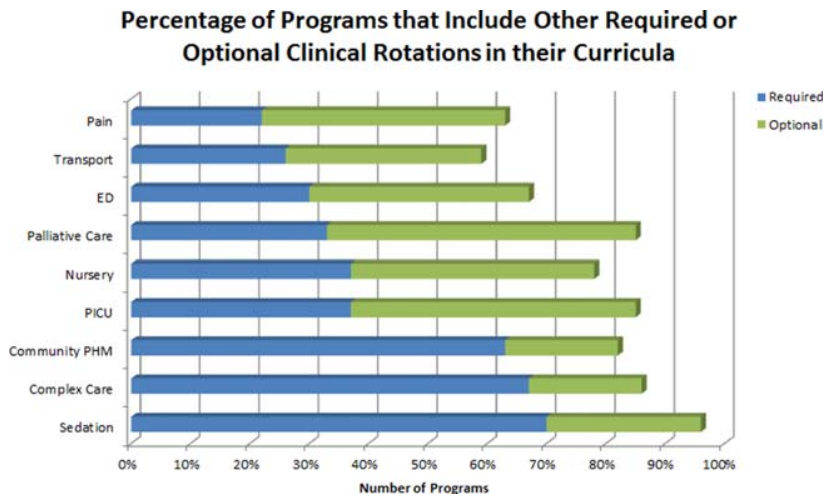

FIG. 2. Percentage of programs that include other required or optional clinical rotations in their curricula. Abbreviations: ED, emergency department; $\mathrm{PHM}$, pediatric hospital medicine; PICU, pediatric intensive care unit.

of weeks on inpatient general pediatrics service by year of training was 15 weeks, 16 weeks, and 16.5 weeks, respectively. In addition to inpatient general pediatrics service time, most programs require other clinical rotations, with sedation, complex care, and inpatient pediatrics at community sites being the most frequent (Figure 2). Of the 6 responding 1-year programs, $5(83 \%)$ allow fellows to bill/generate clinical revenue at some point during their training. Of the 15 responding 2-year programs, $11(73 \%)$ allow fellows to bill/generate clinical revenue at some point during their training. Of the 4 responding 3-year programs, $2(50 \%)$ allow their fellows to bill/generate clinical revenue at some point during their training.

\section{Fellow Scholarly Activities}

With respect to time dedicated to research, the majority of programs offer coursework such as courses for credit, noncredit courses, or certificate courses. In addition, 11 programs offer fellows a masters' degree in areas including public health, clinical science, epidemiology, education, academic sciences, healthcare quality, clinical and translational research, or health services administration. The majority of these degrees are paid for by departmental funds, with tuition reimbursement, university support, training grants, and personal funds making up the remainder. Twenty-one $(81 \%)$ programs provide a scholarship oversight committee for their fellows. Current fellows' $(\mathrm{n}=63)$ primary areas of research are varied and include clinical research $(36 \%)$, quality-improvement research $(22 \%)$, medical education research $(20 \%)$, health services research $(16 \%)$, and other areas $(6 \%)$.

\section{DISCUSSION}

This is the most comprehensive description of pediatric hospital medicine fellowship curricula to date. Understanding the scope of these programs is an important first step in developing a standardized curriculum that can be used by all. The results of this survey indicate that although there is variability among PHM fellowship curricular content, several common themes exist.
The number of clinical weeks on the inpatient general pediatrics service varied from program to program, though the majority of programs require fellows to spend 15 to 16 weeks each year of training. The variability may be due in part to the way in which respondents defined the term "week on clinical service." For example, if the fellow is primarily on a shift schedule, then he/she may only work 2 to 3 shifts in 1 week, which may have been viewed similarly to daily presence on a more traditional inpatient teaching service with 5 to 7 consecutive days of service. The current study did not explore the details of inpatient general pediatric clinical activities or exposure to opportunities to hone procedural skills, areas that are worth investigating as we move forward to better understand the needs of trainees.

Most residency training programs in general pediatrics require a significant amount of inpatient clinical time, specifically a minimum of 10 "units" or months, though only half of this time is required to be in inpatient general pediatrics. $^{8}$ Although non-fellowship trained early career hospitalists may feel adequately prepared to manage the clinical care of some hospitalized children, perceived competency is significantly lower than their fellowship-trained colleagues with regard to care of the child with medical complexity and technologydependence, and with regard to provision of sedation for procedures. ${ }^{7}$ The majority of FDs surveyed in our study indicated that additional clinical experience with sedation, complex care, and inpatient pediatrics at community sites were required of their fellows. Of note, many of these rotations are not commonly required in pediatric residency training programs; however, the PHM core competencies suggest that hospitalists should demonstrate proficiency in these areas to provide optimal care for hospitalized children. Our results suggest that current PHM fellowship curricula help address these clinical gaps. The requirement of these particular specialized experiences may reflect the clinical scope of practice that is expected from potential employers or may be related to staffing needs. It is well documented that the inpatient demographic of large pediatric tertiary care referral centers has changed over the past decade, with an increasing prevalence of children with medical complexity. ${ }^{9,10}$ In both tertiary referral centers and community hospitals, the expansion of the role of the hospitalist in providing specialized clinical services, such as sedation or surgical comanagement, has been significantly driven by financial factors, though a more recent focus on improvement of efficiency and quality of care within the hospital system has relied heavily on hospitalist input. ${ }^{11-13}$ Important next steps in curriculum standardization include ensuring that training programs allow for adequate clinical exposure and proper assessment of competency in these areas, and determining the full complement of clinical training experiences that will produce hospitalists with a well-defined scope of practice that adequately addresses the needs of hospitalized children. 
Most fellowship-trained hospitalists work primarily in university-affiliated institutions with expectations for scholarly productivity. ${ }^{5,7}$ Fellowship-trained hospitalists have made large contributions to the growing body of PHM literature, specifically in the realms of medical education, healthcare quality, clinical pediatrics, and healthcare outcomes. ${ }^{4}$ Many PHM fellowship-trained hospitalists have educational or administrative leadership roles. ${ }^{2}$ Our results indicate that current PHM fellows continue to be active in a variety of research activities. In addition, FDs reported that the vast majority of programs included scholarship oversight committees, which ensure a mentored and structured research experience. Finally, most programs require or offer additional coursework, and many programs with university affiliations allow for attainment of graduate degrees. Inclusion of robust research training and infrastructure in all programs is a paramount goal of PHM fellowship training. This will allow graduates to be successful researchers, generating new knowledge and supporting the provision of high-quality, evidence-based, and value-driven care for hospitalized children.

A unique feature of several PHM fellowship programs is that fellows are allowed to bill for clinical encounters. Many programs rely on clinical revenue to support fellow salaries. ${ }^{14}$ For some programs, a portion of this clinical revenue comes from fellows billing for clinical encounters. ${ }^{15}$ Programs that allow fellows to bill/generate clinical revenue have fellows working in attending roles without direct supervision, whereas nonbilling fellows have direct supervision by an attending. ${ }^{15}$ In the current ABP training model, subspecialty fellows cannot independently bill for clinical encounters within their own subspecialty, though they can moonlight as long as they meet the duty hour requirements set forth by the ACGME. ${ }^{16}$ FDs will need to consider the impact of this requirement on fellow autonomy and on financial revenue for funding fellow salaries if the field achieves ABP subspecialty status.

Regardless of whether or not PHM becomes a designated subspecialty of the ABP, FDs will continue to work together to develop a standard core curriculum that incorporates elements of clinical and nonclinical training to ensure that graduates not only provide high-quality care for hospitalized children, but also generate new knowledge that advances the field in care delivery and quality of care in any setting. The results of this study will not only help to inform curriculum standardization, but also assessment and evaluation methods. Currently, PHM FDs meet annually and are nearing consensus on a standard 2-year curriculum based on the PHM Core Competencies that incorporates core clinical, systems, and scholarly domains. We continue to solicit the input of stakeholders, including new FDs, community hospitalist leaders, internal medicine-pediatrics hospitalist leaders, the Joint Council of Pediatric Hospital Medicine, and leaders of national organizations, such as the American Academy of Pediatrics, Academic Pediatrics Association, and Society of Hospital Medicine. Additional work around standardizing the fellowship application and recruitment process has resulted in our recent acceptance into the Fall Subspecialty Match through the National Residency Match Program, as well as development and implementation of a common fellowship application form. The FD group has recently formalized, voting into place an executive steering committee, which is responsible for the development and execution of long-term goals that include finalizing a standardized curriculum, refining program and fellow assessment methods through critical evaluation of fellow metrics and outcomes, and standardization of evaluation methods.

Adopting a standard 2-year curriculum may affect some programs, specifically those that are currently 1 year in duration. These programs would need to extend the length of their fellowship to allow for the breadth of experiences expected with a standardized 2-year curriculum. This could result in significant financial challenges, effectively increasing the cost to administer the program. In addition, at present, programs have the flexibility to highlight individual areas of strength to attract candidates, allowing fellows to gain an in-depth experience in domains such as clinical research, quality improvement, medical education, or health services research. With a standardized curriculum, some programs may have to assemble specific clinical and nonclinical experiences to meet the agreed-upon expectations for PHM fellowship training. If these resources are not available, programs may need to seek relationships with other institutions to complete their offerings, a possibility that is being actively explored by this group. FDs continue to work with each other to share resources, identify training opportunities, and partner with each other to ensure that the requirements of a standard curriculum can be met.

This study has several limitations. First, it was a voluntary survey of program directors, and though we captured over $80 \%$ of programs at the time of the survey, there are currently more programs that have come into existence and more still that are in the development stage, leading to potential sampling error. Second, variable effort or accuracy by participants may have led to some degree of response error, such as content error or nonreporting error. Third, the survey questions focused on highlevel information, making it difficult to make nuanced comparisons between curricular elements or determine best curricular practice. In addition, this survey did not explore medical education and quality improvement activities of fellows, 2 major areas in which hospitalists play a major role in the inpatient setting. ${ }^{1,17-20}$

\section{CONCLUSION}

PHM fellowship programs have grown and continue to grow at a rapid rate. Variability in training is evident, both in clinical experiences and research experiences, though several common elements were identified in this study. The majority of programs are 2 years, and clinical experience comprises approximately $50 \%$ of training 
time, often including key rotations such as sedation, complex care, and rotations at community hospitals. Future directions include standardizing clinical training and expectations for scholarship, formulating appropriate methods for assessment of competency that can be used across programs, and seeking sustainable sources of funding.

Disclosure: Nothing to report.

\section{References}

1. Freed GL, Dunham KM. Characteristics of pediatric hospital medicine fellowships and training programs. J Hosp Med. 2009;4(3):157-163.

2. Heydarian C, Maniscalco J. Pediatric hospitalists in medical education: current roles and future directions. Curr Probl Pediatr Adolesc Health Care. 2012;42(5):120-126.

3. Bekmezian A, Teufel R, Wilson K. Research needs of pediatric hospitalists. Hosp Pediatr. 2011;1(1):38-44.

4. Oshimura J, Bauer BD, Shah N, Maniscalco J. Pediatric hospital medicine fellowships: outcomes and future directions. Paper presented at: Pediatric Hospital Medicine 2014; July 26, 2014; Orlando, FL.

5. Teufel R, Bekmezian A, Wilson K. Pediatric hospitalist research productivity: predictors of success at presenting abstracts and publishing peerreviewed manuscripts among pediatric hospitalists. Hosp Pediatr. 2012; 2(3):149-160.

6. Stucky ER, Ottolini MC, Maniscalco J. Pediatric hospital medicine core competencies: development and methodology. J Hosp Med. 2010;5:339-343.

7. Librizzi J, Winer J, Banach L, Davis A. Perceived core competency achievements of fellowship and non-fellowship early career pediatric hospitalists. J Hosp Med. 2015;10(6):373-389.

8. Accreditation Council of Graduate Medical Education. ACGME program requirements for graduate medical education in pediatrics.
Available at: https://www.acgme.org/acgmeweb/Portals/0/PFAssets/ 2013-PR-FAQ-PIF/320_pediatrics_07012013.pdf. Published September 30, 2012. Accessed July 7, 2015.

9. Burns KH, Casey PH, Lyle RE, Bird TM, Fussell JJ, Robbins JM. Increasing prevalence of medically complex children in US hospitals. Pediatrics. 2010;126(4):638-646.

10. Simon TD, Berry J, Feudtner C, et al. Children with complex chronic conditions in inpatient hospital settings in the United States. Pediatrics. 2010;126(4):647-655.

11. Sehgal N, Wachter R. The expanding role of hospitalists in the United States. Swiss Med Wkly. 2006;136:591-596.

12. Simon TD, Eilert R, Dickinson LM, Kempe A, Benefield E, Berman S. Pediatric hospitalist comanagement of spinal fusion surgery patients. J Hosp Med. 2007;2(1):23-30.

13. Turmelle M, Moscoso L, Hamlin K, Daud Y, Carlson D. Development of a pediatric hospitalist sedation service: training and implementation. J Hosp Med. 2012;7(4):335-339.

14. Rhim H, Shah N. Sources of funding and support for pediatric hospital medicine fellowship programs. Poster presented at: Pediatric Hospital Medicine 2014; July 27, 2014; Orlando, FL.

15. Council of Pediatric Hospital Medicine Fellowship Directors. Pediatric Hospital Medicine Fellowship Directors Annual Meeting: funding and return on investment. July 24, 2014.

16. Accreditation Council of Graduate Medical Education. Frequently asked questions: ACGME common duty hour requirements. Available at: https://www.acgme.org/acgmeweb/Portals/0/PDFs/dh-faqs2011.pdf. Updated June 18, 2014. Accessed July 7, 2015.

17. Freed G, Duham K. Pediatric hospitalists: training, current practice and career goals. J Hosp Med. 2009;4(3):179-186.

18. Bellet P, Wachter R. The hospitalist movement and its implications for the care of hospitalized children. Pediatrics. 1999;103:473-477.

19. Ottolini M. Pediatric hospitalists and medical education. Pediatr Ann. 2014;43(7):e151-e156

20. Simon T, Starmer A, Conway P, et al. Quality improvement research in pediatric hospital medicine and the role of the Pediatric Research in Inpatient Settings (PRIS) network. Acad Pediatr. 2013;13(6): S54-S60. 